\title{
THE RELATIONSHIP BETWEEN ACUTE RHEUMATISM AND STREPTOCOCCAL ANTIFIBRINOLYSIN
}

\author{
BY \\ C. BRUCE PERRY, M.D., F.R.C.P. \\ Professor of Medicine, University of Bristol
}

That haemolytic streptococci of human origin produce a substance capable of liquefying human fibrin was first demonstrated by Tillet and Garner in 1933. The fibrin from patients recently recovered from haemolytic streptococcal infection was in many cases found to be resistant to the action of this fibrinolysin.

Further work (Tillett, Edwards and Garner, 1934 ; Tillet, 1935) amplified and confirmed these preliminary observations, and demonstrated antifibrinolysin in the blood of about seventy-five per cent. of patients recovering from acute streptococcal infection. The time of appearance of antifibrinolysin in the blood varies in different patients and in different types of infection. Thus Tillett found that in some cases it was present at the time of recovery, but that in others its appearance was delayed for two to four weeks after the acute infection. There was a suggestion that it developed earlier in cases of erysipelas than in those with an acute streptococcal pharyngitis. These observations were confirmed by Myers, Keefer and Holmes (1935), who also showed that the resistance of the plasma to fibrinolysin, once developed, persisted for a varying time, and Spink and Keefer (1936) found that in erysipelas antifibrinolysin can be demonstrated from eight to a hundred and fifty days after the attack. Waaler (1936) found that patients with scarlatina did not all develop antifibrinolysin, and that in those who did, it might first appear from one to five weeks after the infection, similar observations being made by Stuart-Harris $(1935, a$ and $b)$. From this work it is now clear that it is rare for antifibrinolysin to be present in the blood unless the patient has comparatively recently experienced a streptococcal infection. The occasional cases to the contrary can be explained by assuming that the antifibrinolysin has persisted long after the infection to which it is due has been forgotten. Antifibrinolysin ranks with antistreptolysin as one of the antibodies produced as part of the immunity mechanism following infections with haemolytic streptococci. The presence of these antibodies in the blood does not necessarily run parallel, and StuartHarris $(1935 b)$ has shown that there is no correlation between antistreptolysin and antifibrinolysin except that when the fibrin is completely resistant to fibrinolysin there is usually some rise in the antistreptolysin titre.

Shortly after Tillett and Garner's original reports, Hadfield, Magee and Perry (1934) found that the fibrin of many patients in the active stage of acute rheumatism was partially or completely resistant to fibrinolysin. This was regarded as further evidence of the frequent occurrence of a haemolytic 
streptococcal infection before the onset of an attack or relapse of acute rheumatism. This observation on the occurrence of antifibrinolysin in acute rheumatism has been confirmed by McEwen, Alexander and Bunim (1935), Myers, Keefer and Holmes (1935), Stuart-Harris (1935), Tillett (1935) and Waaler (1936).

It therefore appeared desirable to determine, as far as possible, the precise relationship of the development of antifibrinolysin to the onset of acute rheumatism. For this purpose a large number of patients with acute rheumatism has been studied during the past three years by repeated observations of the antifibrinolysin content of the blood during acute attacks of rheumatism, during convalescence, and following upper respiratory infections with haemolytic streptococci.

\section{Method of investigation}

The method used has been essentially that described by Tillett and Garner (1933) and described in the paper reporting the original observations of Hadfield, Magee and Perry (1934). In order to obviate the danger of the organisms losing their fibrinolytic power on subculture, tests on every plasma were made using four different strains of organisms of known fibrinolytic activity and when, on subculture, any of these strains lost its power to produce adequate amounts of fibrinolysin it was replaced by fresh fibrinolytic strains. Two strains, both isolated from cases of puerperal septicaemia and kindly provided by Dr. L. Colebrook, have retained their fibrinolytic activity throughout the period of study despite repeated subculture. As a further check known susceptible plasma was retested with each fresh batch examined. One of the difficulties in working with fibrinolysin is the absence of any standard or numerical titre such as that devised by Todd for streptolysin estimations. An attempt has been made to indicate antifibrinolysin content of the blood by giving those plasmas which were completely resistant to fibrinolysin after twenty-four hours' incubation an arbitrary value of six, and those in which complete liquefaction took place in less than one hour a value of one. The intermediate values are shown in the following table :-

\begin{tabular}{|c|c|c|c|c|c|}
\hline \multicolumn{3}{|c|}{ CLOT } & & $\begin{array}{c}\text { ANTIFIBRINOLYSIN } \\
\text { VALUE }\end{array}$ & SIGNIFICANCE \\
\hline Less than 1 hour & $\cdots$ & $\cdots$ & $\cdots$ & 1 & Within normal limits. \\
\hline 1 to 4 hours .. & . & . & $\cdots$ & 2 & , \\
\hline 4 to 8 hours $\ldots$ & . & . & $\cdots$ & 3 & Doubtful. \\
\hline 8 to 24 hours . & $\because$ & $\cdots$ & . & 4 & Definite increase in antifibrinolysin. \\
\hline $\begin{array}{l}\text { Partial liquetactio } \\
\text { No liquefaction in }\end{array}$ & n 22 & ours & $\cdots$ & 3 & Marked increase in antifibrinolysin. \\
\hline ction & & & . & 6 & , \\
\hline
\end{tabular}

\section{Results}

A considerable number of the records had to be discarded since during the period under consideration the patients suffered no rheumatic relapse and had no acute haemolytic streptococcal infection. Further patients were excluded as they were not seen until comparatively late in the rheumatic attack, and it is now clear that although in many cases antifibrinolysin, once it has developed 
may persist for a considerable time, yet in others it only lasts for one or two weeks. For this reason only those cases of acute rheumatism that came under observation early in the attack have been included in this study. The patients which thus proved suitable for consideration may be divided into four groups.

GROUP A.-This is composed of forty-four cases of acute rheumatism and carditis observed in fifty-seven acute episodes. Nine patients had two attacks and two had three. Thirty-three of these forty-four patients showed resistance to fibrinolysin during the acute stage. In ten antifibrinolysin was present in two attacks (fig. 1) and in the two with three attacks antifibrinolysin was present in all. One patient seen early and right through one attack showed no resistance to fibrinolysin, yet in a subsequent attack this was well marked but lasted less than a month. Thus in fifty-seven attacks of rheumatism in

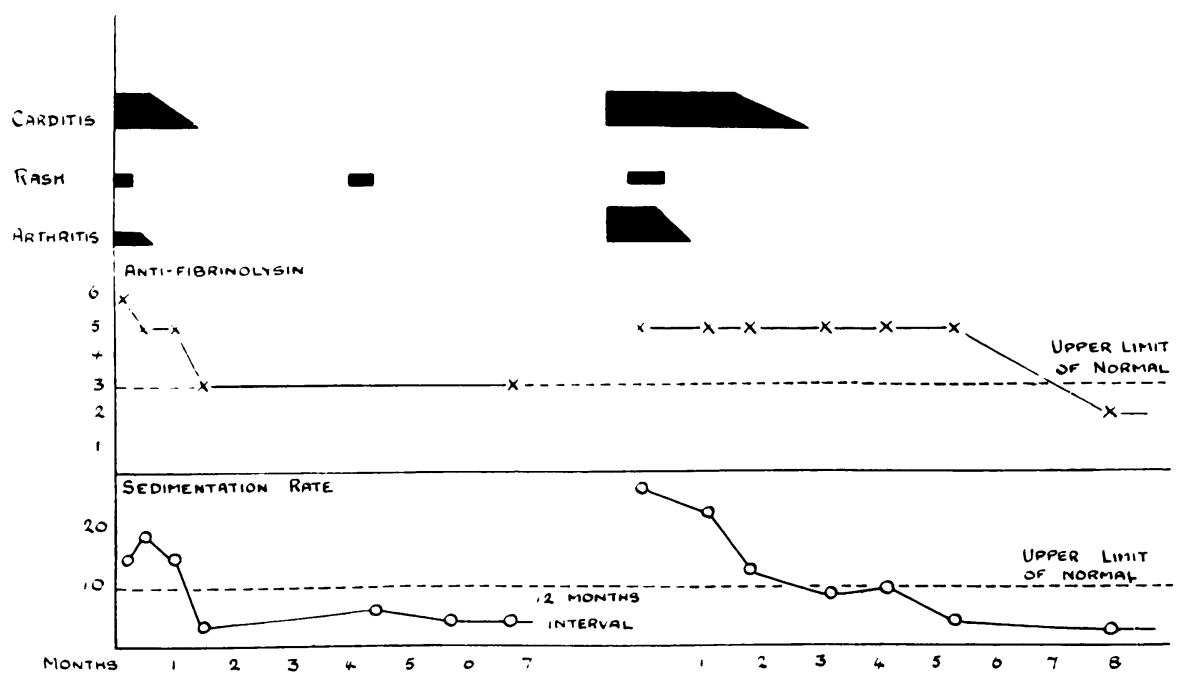

FIG. 1.-A patient observed in two attacks of rheumatism. In both, antifibrinolysin was marked, but in the first it disappeared as the rheumatic activity subsided and in the second persisted long after all activity was at an end.

these forty-four patients, antifibrinolysin was present in forty-five. No obvious difference could be detected in those who showed antifibrinolysin and those in whom this was absent (fig. 2). In one patient with a severe attack of carditis antifibrinolysin was present at the onset of the attack but in the most severe stage of the disease this disappeared only to return as the general condition improved and then lasted for three months, long after all rheumatic activity had subsided (fig. 3). In most instances, resistance to fibrinolysin was most marked at the onset of the rheumatic infection, but in one full resistance did not develop until about a month later. Stuart-Harris $(1935 a)$ found that in patients with acute rheumatism antifibrinolysin persisted after the sedimentation rate was normal and other signs of activity had returned to normal. Whilst this occurred in fourteen attacks, in ten the antifibrinolysin returned to normal before the active rheumatic infection had subsided and in the remainder the antifibrinolysin disappeared as the sedimentation rate fell to normal. Thus 


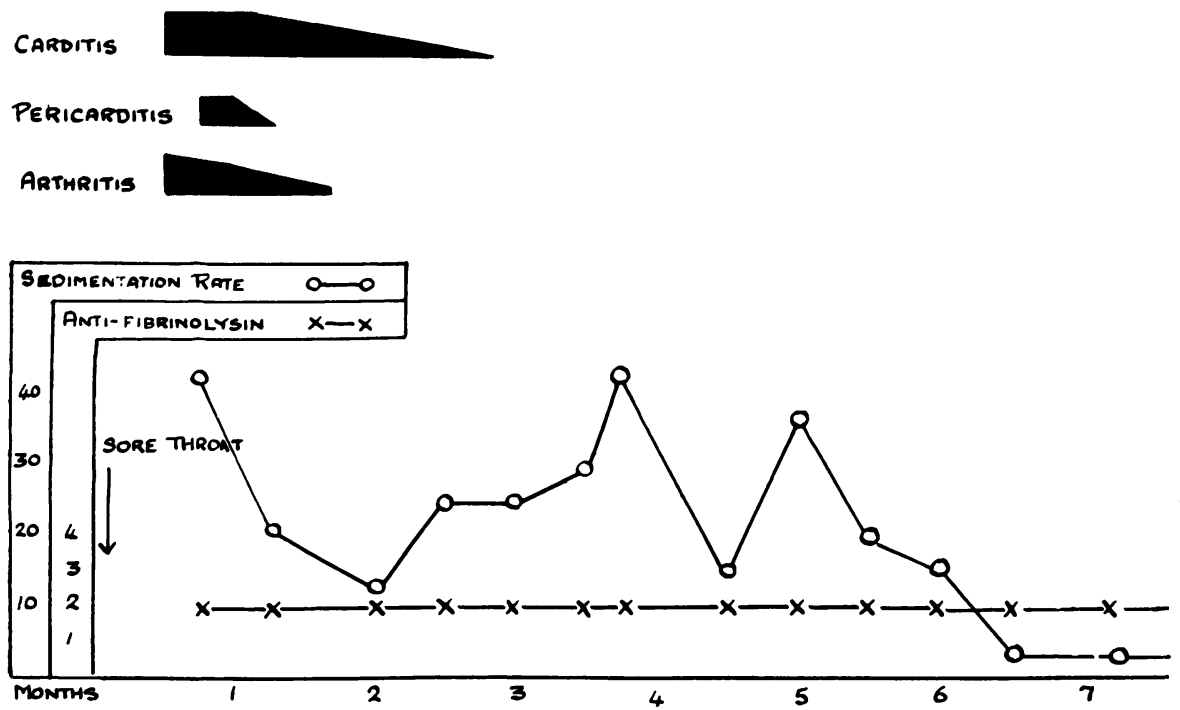

FIG. 2.-A patient with a severe polycyclic attack of acute rheumatism following a sore throat with no rise in antifibrinolysin throughout.

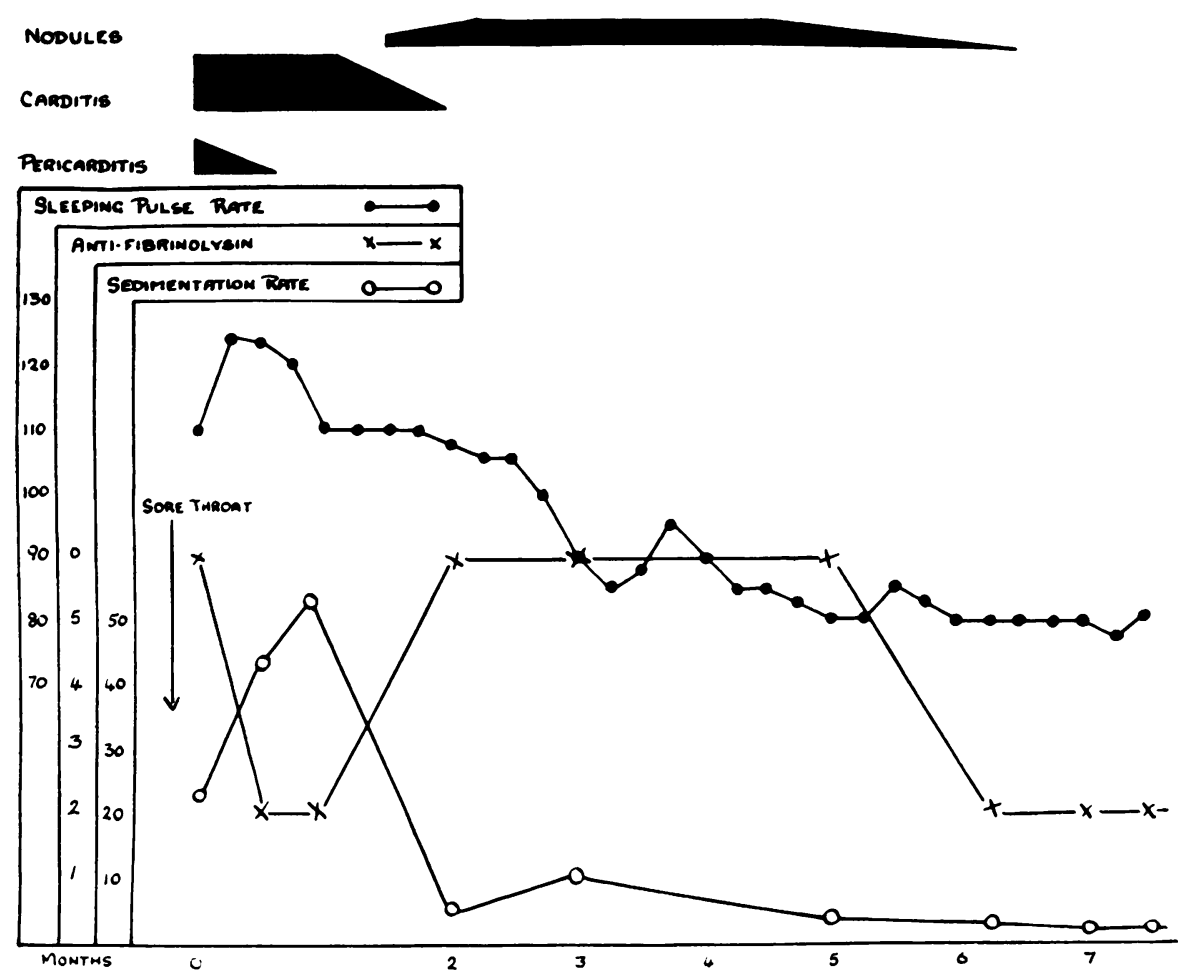

FIG. 3.-A patient with a severe attack of acute rheumatism with depression of the antifibrinolysin titre during the most acute stage of the disease. 
of these forty-four cases seventy-eight per cent. showed resistance to fibrinolysin in the acute phase of the disease. This is similar to the seventy-five per cent. of normal people developing antifibrinolysin following uncomplicated streptococcal infection (Tillett, 1935) and is thus strong presumptive evidence that attacks of acute rheumatism are preceded, in the majority of cases, by a haemolytic streptococcal infection even if this is unrecognized. However, it is also clear that the development of antifibrinolysin is not an essential part of the rheumatic process even in the same child (fig. 4).

GROUP B.-This is a small group consisting of six patients with chorea but no carditis. Of these only one showed antifibrinolysin at the onset of the chorea and this lasted for less than a month. Although the number of cases

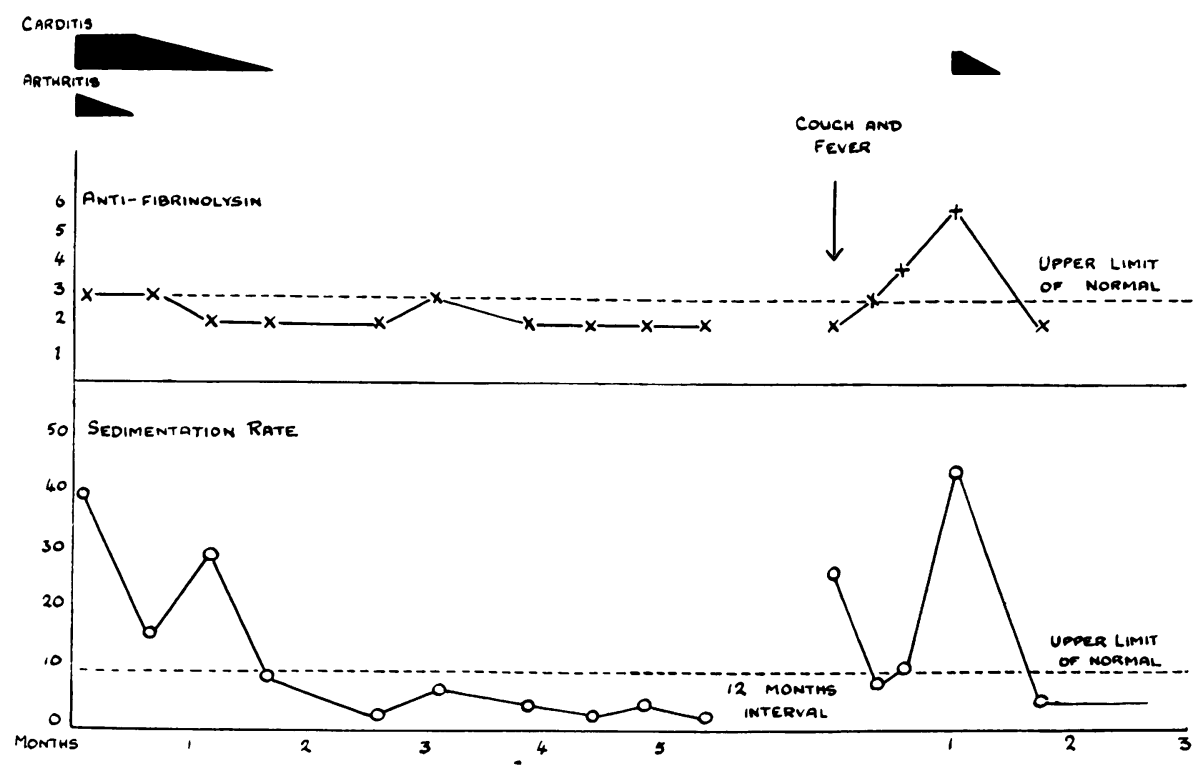

FIG. 4.-A patient with a severe attack of arthritis and carditis showed no definite antifibrinolysin, but in a subsequent attack twelve months later this was well marked.

in this group is small, it appears to indicate that chorea is less frequently preceded by a haemolytic streptococcal infection than are the other rheumatic manifestations. This agrees with clinical observations (Perry, 1938).

GROUP C.-This consists of fifteen patients, fourteen with chronic but quiescent rheumatic heart disease and one old case of chorea. While under observation these children developed a haemolytic streptococcal sore throat. There was no definite recurrence of rheumatic infection, but five of the fourteen patients with carditis showed a rise in sleeping pulse rate, which lasted from seven to twenty-one days and which occurred fourteen days to two months after the sore throat. During this time the sedimentation rate was normal and no other evidence of activity was detected (fig. 5). Of the fifteen cases only two developed antifibrinolysin following the attack. In one this appeared three weeks after the sore throat but was not present when the blood was next 
examined fourteen days later. The other patient showed resistance to fibrinolysin eleven days after the sore throat and this persisted for two months. The remaining thirteen, including the case of chorea and the five cases showing a rise in the sleeping pulse rate, developed no resistance to fibrinolysin (fig. 5). In three of these resistance had been well marked in the antecedent attack of rheumatism, as was also the case of the child with chorea. All of these fifteen patients received aspirin (thirty grains daily) for six weeks following the sore throat in an attempt to prevent a relapse of rheumatism (Schlesinger, 1937).

GROUP D.-This is composed of ten children with carditis who were observed during a quiescent period and who developed a haemolytic streptococcal sore throat followed by a rheumatic relapse. Six developed resistance

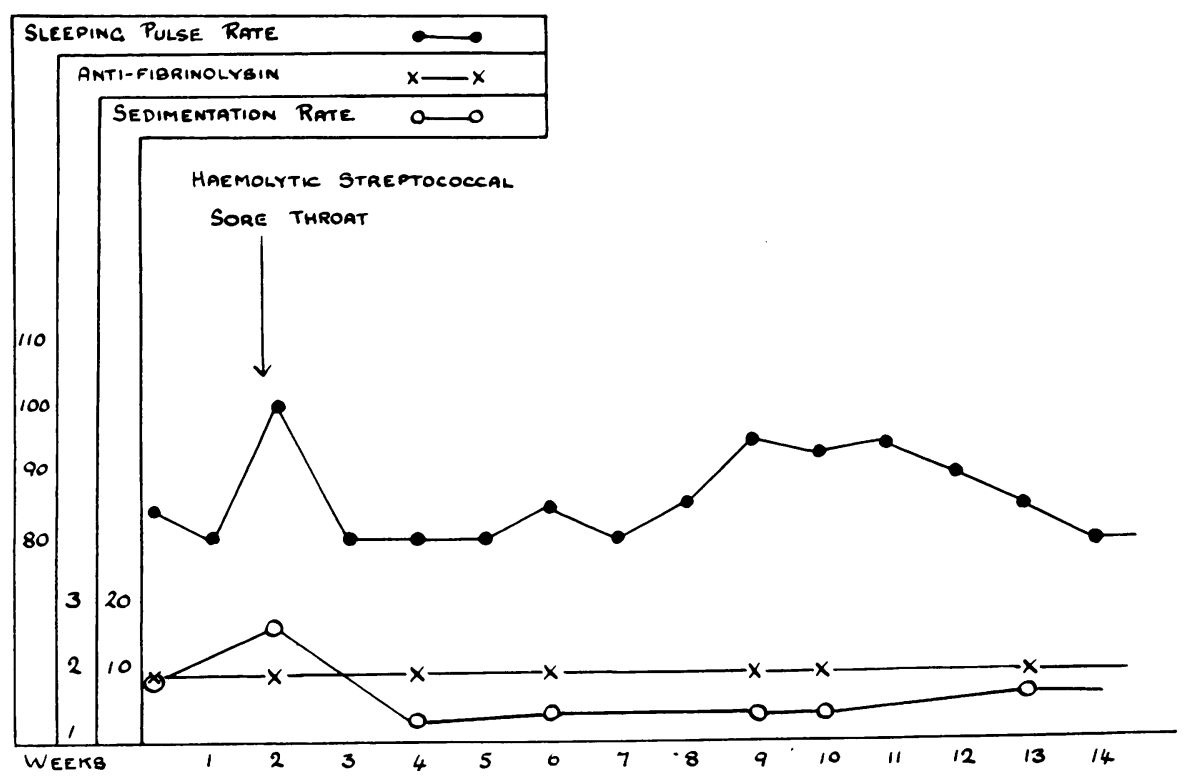

FIG. 5.-A patient with a streptococcal sore throat following which there was no rise in antifibrinolysin and no relapse. (Patient received aspirin for six weeks following the sore throat.)

to antifibrinolysin at the same time as the attack of rheumatism, which was severe in all six and in two fatal (fig. 6). Four failed to show antifibrinolysin, the rheumatic relapse in all four being mild. These four patients received aspirin, whereas only two of the patients in which antifibrinolysin appeared were given it. These findings, especially when taken in conjunction with results found in group C, are at striking variance with Stuart-Harris's observation that resistance to fibrinolysin developed with marked frequency in rheumatic children following a streptococcal sore throat. Schlesinger (1937) has suggested that the administration of salicylates to rheumatic children following a haemolytic streptococcal sore throat tends to prevent relapses. Whether this view is correct or not, the present study indicates that it does tend to inhibit antifibrinolysin formation. This observation agrees with those of Derrick, 
Hitchcock and Swift (1928) who found that the administration of salicylates to patients who had received horse serum tended to prevent the development of anti-horse serum precipitin and the arthritis of serum sickness. This possible action of the salicylates in inhibiting antibody formation merits further investi-

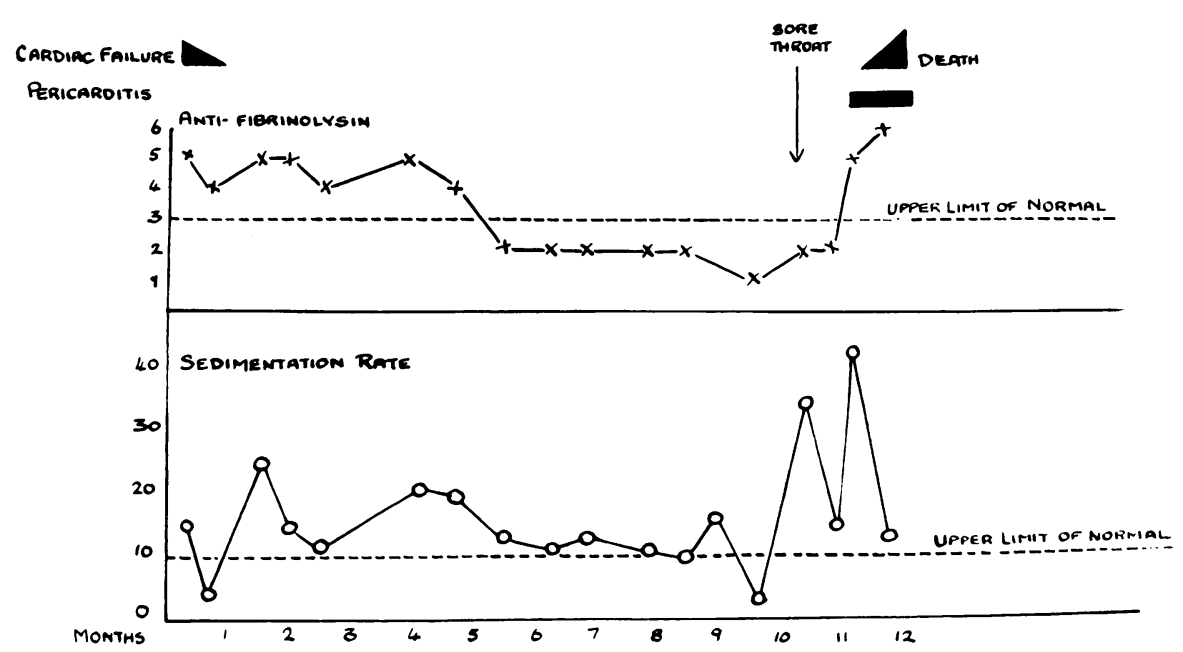

FIG. 6.-A patient with a severe attack of carditis with failure who subsequently developed a streptococcal sore throat followed by a recurrence of carditis which proved fatal. Antifibrinolysin was present in both attacks. (No aspirin.)

gation. Groups $\mathrm{C}$ and $\mathrm{D}$ thus show that although the majority of patients with a relapse of acute rheumatism following a streptococcal infection show resistance to fibrinolysin, this is not invariably the case. Further, rheumatic patients who develop antifibrinolysin following a streptococcal infection do not necessarily develop a rheumatic relapse.

\section{Conclusions}

From this investigation it is clear that the appearance of resistance to streptococcal fibrinolysin in the blood of patients with acute rheumatism is not an essential part of the rheumatic process. Antifibrinolysin is present in about seventy-eight per cent. of patients with acute rheumatism, which is approximately the proportion of normal patients who develop antifibrinolysin following a streptococcal infection. There is no correlation between the duration of antifibrinolysin and the duration of the rheumatic attack or between antifibrinolysin and the severity of the rheumatic attack. There is some evidence that the administration of salicylates following a streptococcal infection inhibits the formation of antifibrinolysin.

Thanks are due to the Colston Research Society of the University of Bristol for a grant which made this investigation possible and also to Mr. W. S. Emery for his technical assistance. 


\section{REFERENCES}

Derrick, C. L., Hitchcock, C. H., and Swift, H. F. (1928). J. clin. Invest., 5, 427.

Hadfield, G., Magee, V., and Perry, C. B. (1934). Lancet, 1, 834.

McEwen, C., Alexander, M. A., and Bunim, J. J. (1935). J. Lab. clin. Med., 21, 465.

Myers, W. K., Keefer, C. S., and Holmes, W. F. (1935). J. clin. Invest., 14, 119.

Perry, C. B. (1938). Proc. Int. Cong. on Rheumatism and Hydrology, London, 134.

Schlesinger, B. (1937). Recent Advances in the Study of Rheumatism, London, 91.

Spink, W. W., and Keefer, C. S. (1936). J. clin. Invest., 15, 21.

Stuart-Harris, C. H. (1935a). Lancet, 2, 1456.

- (1935b). Brit. J. exp. Path., 16, 513.

Tillet, W. S. (1935). J. clin. Invest., 14, 276.

- and Garner, R. L. (1933). J. exp. Med., 58, 485.

- Edwards, L. B., and Garner, R. L. (1934). J. clin. Invest., 13, 47.

Waaler, E. (1936). Norsk. Mag. Laegevidensk., 97, 449. 